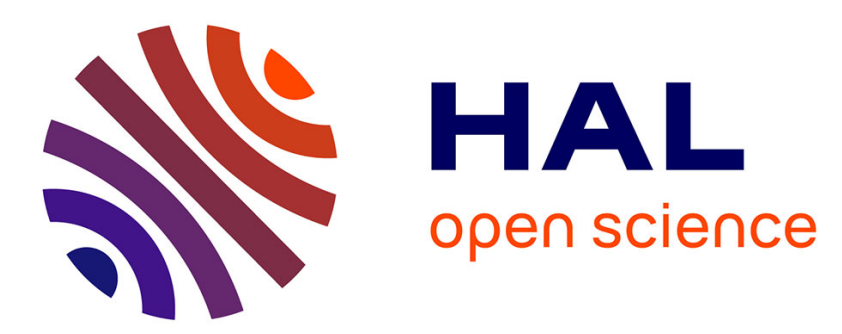

\title{
Phenomenological model of preloaded spindle behavior at high speed
}

Clément Rabréau, David Noel, Sébastien Le Loch, Mathieu Ritou, Benoît

Furet

\section{- To cite this version:}

Clément Rabréau, David Noel, Sébastien Le Loch, Mathieu Ritou, Benoît Furet. Phenomenological model of preloaded spindle behavior at high speed. International Journal of Advanced Manufacturing Technology, 2017, 90 (9-12), pp.3643 - 3654. 10.1007/s00170-016-9702-1 . hal-01819016

\section{HAL Id: hal-01819016 https://hal.science/hal-01819016}

Submitted on 19 Jun 2018

HAL is a multi-disciplinary open access archive for the deposit and dissemination of scientific research documents, whether they are published or not. The documents may come from teaching and research institutions in France or abroad, or from public or private research centers.
L'archive ouverte pluridisciplinaire HAL, est destinée au dépôt et à la diffusion de documents scientifiques de niveau recherche, publiés ou non, émanant des établissements d'enseignement et de recherche français ou étrangers, des laboratoires publics ou privés. 


\title{
Phenomenological model of preloaded spindle behavior at high speed
}

\author{
Clément Rabréau · David Noël · Sébastien Le Loch • Mathieu Ritou • \\ Benoit Furet
}

Received: date / Accepted: date

\begin{abstract}
High Speed Machining spindles are high precision mechanisms with a complex and very sensitive behavior. Frequency Response Functions are required to avoid unstable cutting conditions that lead to premature failure of spindle and tool. However, FRFs are affected by stiffness loss of the bearings at high speed. Indeed, the rotor's behavior is driven by its boundary conditions which are the preloaded bearings. In order to obtain an accurate model of the preloaded bearing system, this paper focuses on the axial spindle behavior. An analytical model that computes the equilibrium state of the shaft, rear sleeve and bearing arrangement is presented. A model enrichment method is presented with several new physical phenomena: the macroscopic deformations of the shaft and bearing rings as well as the rear sleeve's complex behavior. The significance of these phenomena is evaluated with a sensitivity analysis and used for the model updating, to obtain a just accurate enough model. The contributions of these enrichments are presented for a case study performed on an industrial spindle. A good agreement between the simulation and the experimental results are achieved that validates the model updating strategy and the phenomenologically enriched model.
\end{abstract}

Keywords spindle dynamics · angular contact ball bearing $\cdot$ model updating $\cdot$ bearing preload

C. Rabréau

IRCCyN (UMR CNRS 6597 Institut de Recherche en Communications et Cybernétique de Nantes),

1 quai de Tourville, 44035 Nantes, France E-mail: clement.rabreau@univ-nantes.fr

\section{Introduction}

HSM spindles are used for the machining of advanced and high value parts, such as aircraft structural parts. A good understanding of the spindle dynamics is required to optimize the machining process and to avoid vibrations that affect the quality of machined parts and the lifetime of the spindle. The simulation approach is of interest to find optimal cutting conditions, compared to long and expensive experiments. Numerical simulation can also lead to a better understanding of the complex phenomena involved in the spindle dynamics.

A complete model to predict the dynamic behavior of spindles is usually composed of a rotating shaft model and of a bearing model. Most of the rotating shaft models found in the literature are based on 1D Finite Element with beam theory: Euler Bernoulli or Timoshenko [5, 9, 16]. 3D Finite Element model can also be found [26].

A major part of HSM spindles is equipped with angular contact ball bearings. They play an important role in the global spindle behavior, especially due to the dynamic effects on balls $[1,5,6,8,15]$. Bearing model gives the relation between the global load and the global displacement of the bearing $[12,21]$ and therefore the bearing stiffness. An accurate bearing stiffness is crucial in a model as it has a great impact on the spindle frequency response function [24]. Five degrees of freedom (DoF) model of bearing that consider dynamic effects on balls were developed by Jones [14]. This bearing model were included in spindle models and lead to interesting results [5]. A new method for the stiffness matrix computation that includes the dynamic effects was presented in [19]. The radial expansion of the bearing ring due to the centrifugal force as well as the thermal effects was added to the model in $[4,26]$. 
Angular contact ball bearings are mounted into spindles with a preload, whose value has a great influence on bearing stiffness [6] and therefore on the spindle behavior [20, 23, 25, 27]. Two types of preload system exist: rigid and elastic ones. The latter is composed of springs used to apply the preload force. Contrary to the rigid preload, it is less sensible to thermal effects and the preload force varies less with speed. They are compared in [3]. It was shown that spindle systems with rigid preload have higher stiffness than systems with elastic preload. However, it is not suitable for hot rotors. Indeed, the motor causes thermally induced preload increase that needs to be controlled to preserve the system $[2,11]$. Elastic preload is therefore widely used in HSM electrospindle, although there is a complex axial behavior of the shaft and of the rear sleeve containing the rear bearings [13].

The thermal effect on the shaft together with other phenomena have also been studied in [10] with the use of a novel multi physic model, designed for an highspeed aerostatic spindle.

In most of the spindle models that can be found in the literature, the bearing stiffness values are obtained from nominal parameters values and with a fixed preload force $[9,22]$. The non-linearity of the bearing behavior was considered in several studies [5, 26], however the complex axial behavior and preload evolution influence was not regarded.

The method introduced in [18] that will be completed in this paper takes into account bearing nonlinearity and also preload variation with speed.

To obtain an accurate model of the spindle dynamics, the complete understanding of the preloaded bearing arrangement is required. Indeed, the rotor's FRFs are well affected by the bearing stiffness loss at high speed. This paper focuses on the study of the axial behavior of a spindle. The objective is to understand and model the behavior of the bearing preloaded system in relation to the spindle speed and to update the model parameters that are of importance in complete spindle models. This research aims at finding the right boundary conditions for dynamic model of spindle (i.e. bearing stiffness and preload forces in relation to spindle speed).

A 5 DoF bearing model is used and lead to a complete stiffness matrix. It is based on Jones work and include the dynamics effects on the balls as well as radial expansions of the rings. The axial equilibrium of the shaft and the rear sleeve is formulated analytically and solved for different axial loads and spindle speeds. Several new physical phenomena are added to the model: the macroscopic deformations of the shaft and bearing rings as well as the rear sleeve's complex behavior. Their formulation and influence are provided. A new updating strategy based with phenomenological enrichment is presented. Significance classification of the phenomena is achieved based on sensitivity analysis. Experimentations that uses a novel loading device used for the model updating are described and the model updating results are compared with the experiments. The added phenomena's importance is discussed as well as the strategy to build a just accurate enough model.

\section{Spindle Model}

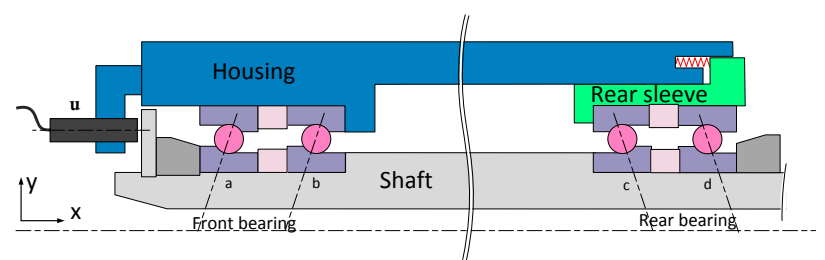

Fig. 1 Bearing arrangement of the Fischer MFW1709 spindle.

The axial model is developed in order to understand the complex behavior of the bearing preloaded system at high speed. It consists of the solving of the axial equilibrium analytical equations. A Fischer electrospindle (MFW1709 - $24000 \mathrm{rpm} 40 \mathrm{~kW}$ ) was considered in this study. The structure of the spindle and the bearing arrangement are presented in Figure 1. It is composed of a spring preloaded back to back tandem arrangement of hybrid ball bearings.

\subsection{Model Principle}

An accurate 5 DoF bearing model is required to construct a numerical model of rotor dynamics. It provides the relation between the global displacement $\mathbf{d}=$ $\left(\delta_{x}, \delta_{y}, \delta_{z}, \theta_{y}, \theta_{z}\right)^{t}$ and the global loads $\mathbf{f}=\left(F_{x}, F_{y}, F_{z}, M_{y}, M_{z}\right)^{t}$ on the inner ring of the bearing as presented in Figure 2 . Therefore, it gives the boundary conditions of the rotor model.

These boundary conditions are expressed as a $5 \times 5$ stiffness matrix $\mathbf{K}$. Since the paper focuses on the axial spindle model, only the axial stiffness $K(1,1)=K_{x x}$ is considered.

A 5-DOF analytical bearing model which consider the dynamic effects on the balls and the macroscopic radial expansions of the rings is considered. Based on 


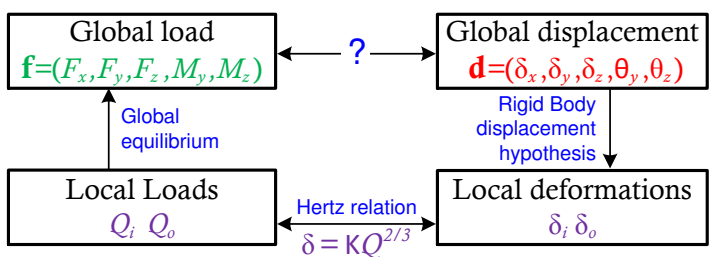

Fig. 2 Bearing model principle [19].

Lynagh [17] work, it can be deduced in [7] that raceway roundness errors are negligible.. Since no explicit expression between $\mathbf{d}$ and $\mathbf{f}$ can be obtained, the local equilibrium of each ball is expressed.

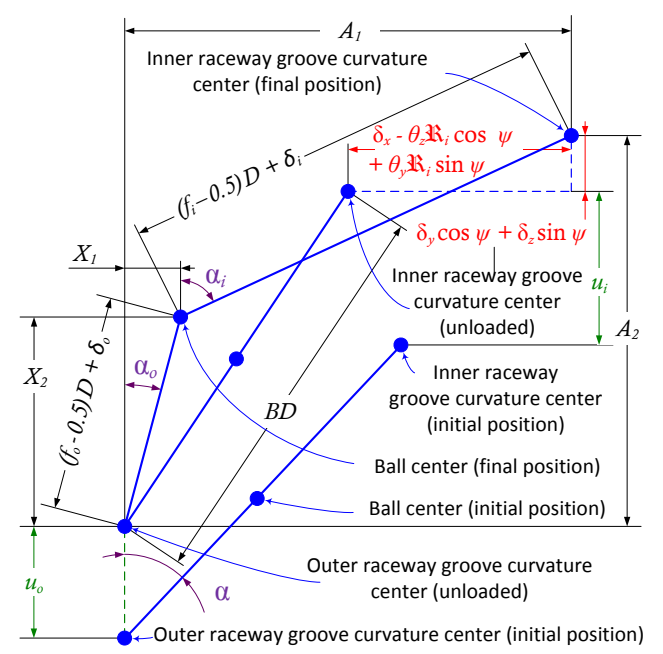

Fig. 3 Position of the ball center and raceways groove curvature centers, with and without ring deformation and load.

The locations of the curvature center of the inner ring, outer ring and ball are shown in Figure 3. The initial positions refer to an unloaded bearing without ring expansion. The unload positions refers to an unloaded bearing with ring expansion. Lastly, the final position refers to a bearing with load and ring expansion. The position of the inner ring and the balls changes due to an external load on the bearing. The distance between the inner raceway groove curvature center before and after loading are respectively projected on the axial and radial directions in equation (1). $\Delta u_{N}=u_{i}-u_{o}$ is the relative radial expansion between the inner and outer rings of the bearing. The macroscopic deformations of the ring are due to thermal and centrifugal effect. Thus, it is assumed to vary with spindle speed $N$. In section 3 and 5 , the rings radial expansion is considered as one of the model enrichments and the "basic model" refers to a 5 DoF bearing model with dynamic effects on balls but without ring radial expansion.

$$
\begin{aligned}
& A_{1}=B D \sin \alpha+\delta_{x}-\theta_{z} \Re_{i} \cos \psi+\theta_{y} \Re_{i} \sin \psi \\
& A_{2}=B D \cos \alpha+\delta_{y} \cos \psi+\delta_{z} \sin \psi+\Delta u
\end{aligned}
$$

Equations (2) are obtained from Figure 3 while applying the Pythagorean theorem.

$$
\begin{aligned}
& \left(A_{1}-X_{1}\right)^{2}+\left(A_{2}-X_{2}\right)^{2}-\left[\left(f_{i}-0.5\right) D+\delta_{i}\right]^{2}=0 \\
& X_{1}^{2}+X_{2}^{2}-\left[\left(f_{o}-0.5\right) D+\delta_{o}\right]^{2}=0
\end{aligned}
$$

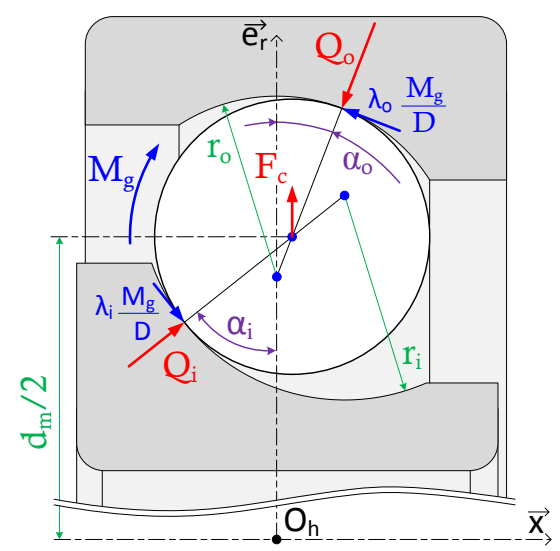

Fig. 4 Dynamic equilibrium of the ball.

The Newton's second law of motion is applied to each ball, see Figure 4. Assuming that contact surfaces can provide sufficient reaction forces to the ball gyroscopic moment $M_{g}$ (i.e. $\lambda_{o} M_{g} / D \leq \mu Q_{0}$ and $\lambda_{i} M_{g} / D \leq$ $\mu Q_{i}$, with $\mu$ the friction coefficient at the contact), it leads to :

$$
\begin{aligned}
& Q_{i} \sin \alpha_{i}-Q_{o} \sin \alpha_{o}+\frac{M_{g}}{D}\left(\lambda_{i} \cos \alpha_{i}-\lambda_{o} \cos \alpha_{o}\right)=0 \\
& Q_{i} \cos \alpha_{i}-Q_{o} \cos \alpha_{o}+\frac{M_{g}}{D}\left(\lambda_{i} \sin \alpha_{i}-\lambda_{o} \sin \alpha_{o}\right)+F_{c}=0
\end{aligned}
$$

$F_{c}$ and $M_{g}$ represents the centrifugal force and respectively the gyroscopic moment on balls. The coefficients $\lambda_{i}$ and $\lambda_{o}$ express the gyroscopic moment distribution. They are determined by the equilibrium of the friction moments at ball/raceway contacts (detailed in [19]). $Q_{i}$ and $Q_{o}$ are the contact forces that are expressed in terms of local displacement $\delta_{i}$ and $\delta_{o}$ with 
the Hertzian theory as : $\delta=K Q^{2 / 3}$. The local variables $\mathbf{x}=\left(X_{1}, X_{2}, \delta_{o}, \delta_{i}\right)$ are used to solve local equilibrium with eq. (2) and (3). The global load $\mathbf{f}$ are then obtained from the sum of the equilibrium contribution of each balls.

$$
\begin{aligned}
F_{x} & =\sum_{z} Q_{i} \sin \alpha_{i}+\lambda_{i} \frac{M_{g}}{D} \cos \alpha_{i} \\
F_{y} & =\sum_{z}\left(Q_{i} \sin \alpha_{i}-\lambda_{i} \frac{M_{g}}{D} \sin \alpha_{i}\right) \cos \psi \\
F_{z} & =\sum_{z}\left(Q_{i} \sin \alpha_{i}-\lambda_{i} \frac{M_{g}}{D} \sin \alpha_{i}\right) \sin \psi \\
M_{y} & =\sum_{z}\left[\Re_{i}\left(Q_{i} \sin \alpha_{i}+\lambda_{i} \frac{M_{g}}{D} \cos \alpha_{i}\right)-\lambda_{i} f_{i} M_{g}\right] \sin \psi \\
M_{z} & =\sum_{z}\left[-\Re_{i}\left(Q_{i} \sin \alpha_{i}+\lambda_{i} \frac{M_{g}}{D} \cos \alpha_{i}\right)+\lambda_{i} f_{i} M_{g}\right] \cos \psi
\end{aligned}
$$

The stiffness matrix $\mathbf{K}$ represent the linearized behavior of the bearing for a given loading state. It is calculated as a Jacobian matrix built from the partial derivatives of load with respect to the displacement : $\mathbf{K}=[\partial \mathbf{f} / \partial \mathbf{d}]$. Noel et al. [19] detailed the analytical computation method that is used to obtain the stiffness matrix.

From the axial stiffness $K_{x x}$ of the bearings, an axial model of the spindle is built based on Figure 1 and 5. The rotor is assumed to be rigid with a quasi-static behavior. The axial displacements $\mathbf{u}=\left(u, u_{p}\right)$ of respectively the shaft and the rear sleeve are calculated for a given axial load $F$ depending on the preload $P$ and preload stiffness $K_{p}$. The model is based on the following equilibrium equations of the shaft and the sleeve

$0=\left(\begin{array}{c}F-F_{1}+F_{2} \\ P-K_{p} u_{p}-F_{2}\end{array}\right)$

$F_{1}$ and $F_{2}$ are the axial loads on the bearing groups that are obtained with the bearing model, see Eq. (6) and Figure 5. As ball bearings are in tandem setup, the load obtained from the model are doubled (i.e. $F_{1}=$ $2 F_{a}=2 F_{b}$, with $a$ and $b$ the two front bearings of the spindle in Figure 1).

$$
\begin{aligned}
& \delta_{x 1}=u+\delta_{x 1,0} \stackrel{\text { analytical bearing model }}{\longrightarrow} F_{a} \\
& \delta_{x 2}=u_{p}-u+\delta_{x 2,0} \stackrel{\text { analytical bearing model }}{\longrightarrow} F_{c}
\end{aligned}
$$

$\delta_{x 1,0}$ and $\delta_{x 2,0}$ correspond to the axial displacement of the ball bearing due to the preload state.

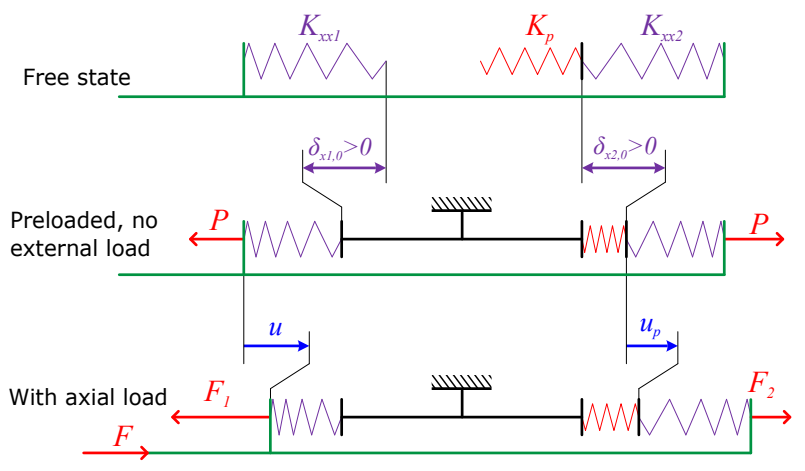

Fig. 5 Spindle axial model.

The equations (5) are solved using the trust region dogleg algorithm of the Matlab fsolve function. This iterative algorithm uses the following Jacobian matrix

$$
\mathbf{J}=\left[\begin{array}{cc}
-K_{x x 1}-K_{x x 2} & K_{x x 2} \\
K_{x x 2} & -K_{p}-K_{x x 2}
\end{array}\right]
$$

This algorithm is chosen because of nonlinearity in the model (see 3.1).

\subsection{Model Enrichment Methodology}

A parameter enrichment methodology is introduced in this paper, in order to simulate and understand the complex and coupled spindle behavior observed experimentally. Indeed, the updating of the above presented model does not match with the experiments (as shown in section 5.1). The method consists in the following steps:

1. Sensitivity analysis to identify the parameters to be updated. Assuming a large set of parameters, a sensitivity analysis brings out the parameters whose variations have the greatest impact on the model.

2. Model updating with the selected parameters.

3 . If the result does not match with experiments, inclusion of new physical phenomena in the model, regarding the previous updating sensitivity analysis results. The high sensitivity of a parameter that has been updated or if the updated value corresponds to a boundary can denote a lack in the modeling or a missing phenomenon. The sensitivity of new parameters describing new phenomena is then added to evaluate their influence.

4. Repeat step 2 and 3 until updating residuals are small enough, so that simulation match with experiments.

Another output of the enrichment methodology based on sensitivity analysis is that a classification of the significance of the physical phenomena in the model is 
obtained. This classification vary from one spindle to another, in relation to their design. It is useful to obtain a good compromise between model simplicity and accuracy.

This methodology ensures that the final enriched model only contains relevant parameters and that the selected physical phenomena have a significant impact on the spindle behavior. These updating procedure can be employed with another objective. If the phenomena that occur are already known, the sensitivity analysis will help to find an order of importance of the phenomena and select the parameter to be included in the model. Therefore, a model as simple as possible but precise enough can be built.

The model updating is achieved with the Matlab fmincon function that minimizes the following cost function (i.e. the error of the shaft displacement) :

$\varepsilon=\sqrt{\frac{1}{i \times j} \sum_{j} \sum_{i}\left(\left.u_{s i m}(i)\right|_{N=j}-\left.u_{e x p}(i)\right|_{N=j}\right)^{2}}$

with $i$ the external load values selected for the update, and $j$ the selected speed values (i.e. 4000, 16000 and 24000rpm).

To perform the sensitivity analysis of the model parameter, a One-Factor-At-a-Time (OFAT) method is used. The variation of the model output is evaluated for an independent variation of each parameter. The other parameters are fixed to their nominal value. All the parameters have not the same dimensions, there are for example force, stiffness and geometric parameters in the model. It is therefore important to adapt the method to be able to compare the different parameters. A variation range parameter $\Delta p_{s}$ is then introduced and selected to be physically possible. The sensitivity $\kappa_{s}$ of the parameter $p_{s}$ is thus expressed by Eq. (9), with $\zeta$, the perturbation fixed at $1 \%$.

$\kappa_{s}=\frac{1}{\zeta}\left[\varepsilon\left(p_{s, n o m}+\zeta \Delta p_{s}\right)-\varepsilon\left(p_{s, n o m}\right)\right]$

\section{Phenomenological enrichment}

The model presented above based on a classical 5 DoF model of bearing failed to predict accurately the spindle axial behavior, as it will be shown in subsection 5.1. Using the parameter enrichment methodology, several physical phenomena have been added to the model. This section explain how these new phenomena are modeled and what are their influences on the axial behavior of the spindle.
In order to understand the influence of the new physical phenomena, Figure 6 presents the evolution of loads on the front bearing $F_{1}$ (solid lines) and rear bearing $F_{2}$ (dashed lines) in case of a rigid and of an elastic preload arrangements, in relation to the axial displacement of the shaft.

Note that Figure 6 also reveals the impact of the dynamic effects on ball, in relation to spindle speed.

\subsection{Limited stroke of the preload system}

The motion of the rear sleeve can be limited by a stop that changes the behavior of the spindle. When the rear sleeve reaches the stroke limit, the spindle turns into a rigidly preloaded configuration, see Figure 7 . Indeed, the presence of the stop adds inflexion points $(B, B$, and $B$ ") on the preloaded bearing evolution curves $F_{2}$ that correspond to an increasing of the axial stiffness of the spindle, especially at lower speed.

To model the stroke limit on the rear sleeve, a new parameter $u_{p, l i m}$ is added to the model to constrain the displacement of the sleeve. At each iteration of the equilibrium resolution algorithm, if $u_{p} \leq u_{p, \text { lim }}$ then, $u_{p}$ is fixed at $u_{p, l i m}$. Therefore, only the equilibrium of the shaft is resolved (see Eq.(5)). A gradually increasing contact surface in the preload system stop can also be considered. It might be due to very low planarity or parallelism defect between sleeve and housing. Assuming an angle $\gamma$ between the two contact surfaces of rear sleeve and the stop, a second parameter, $\Delta s l$ (= $\tan \gamma \times$ contact diameter) is established. The gradually increasing contact surface is modeled as an increasing stiffness $K_{s l}$, see Eq. (10). The additive $K_{s l}$ stiffness is equal to 0 before the contact when $u_{p} \geq u_{p, l i m}+\Delta s l$, and tends to infinity when $u_{p}$ approaches $u_{p, l i m}$ (which is the case for rigid preload).

$$
K_{s l}= \begin{cases}0 & \text { if } u_{p} \geq u_{p, l i m}+\Delta s l \\ \tan ^{2}\left(\frac{\pi}{2} \cdot \frac{\Delta s l+u_{p, l i m}-u_{p}}{\Delta s l}\right) & \text { if } u_{p, l i m}<u_{p}<u_{p, l i m}+\Delta s l \\ \infty & \text { if } u_{p} \leq u_{p, \text { lim }}\end{cases}
$$

The stroke limit stiffness is added to the spring stiffness in the axial equilibrium equation $\left(K_{p}\right.$ is replaced by $K_{p}+K_{s l}$ in Eq. (5). A new corresponding Jacobian $J$ matrix is therefore used in the solving algorithm when $u_{p, l i m}<u_{p}<u_{p, l i m}+\Delta s l$ (see A). 


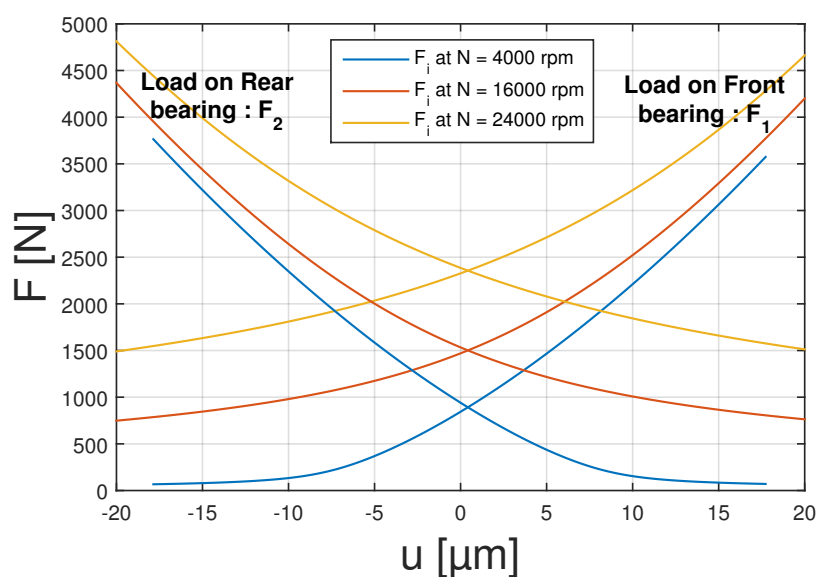

(a) Load on the bearings with a rigid preload

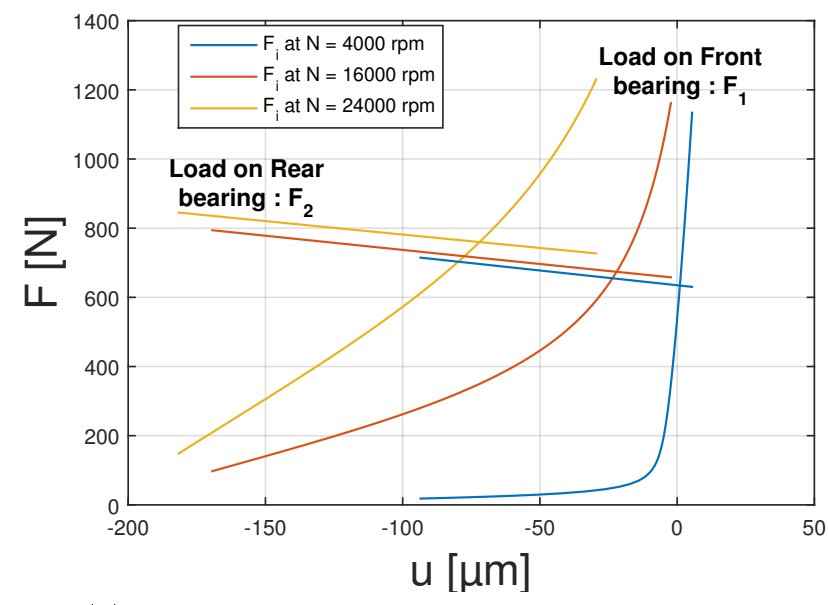

(b) Load on the bearings with an elastic preload

Fig. 6 Comparison of the behavior of rigid preload and elastic preload systems.

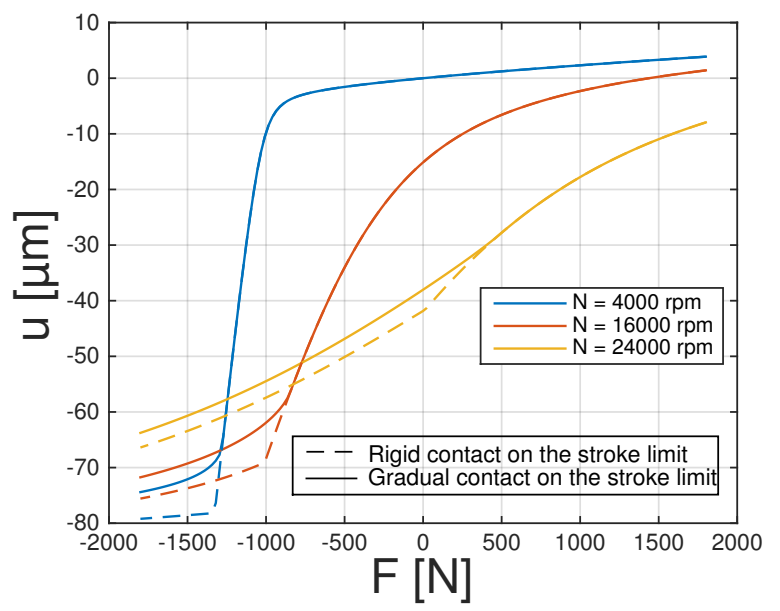

(a) Axial displacement of the shaft

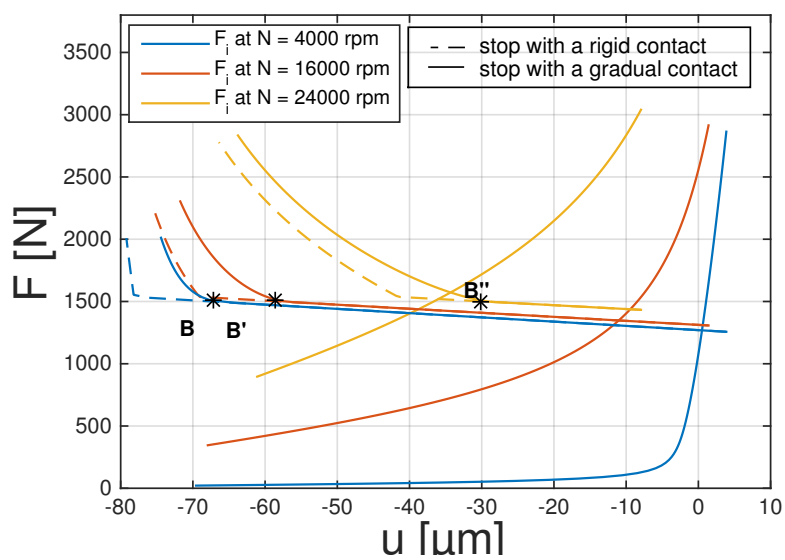

(b) Loads on the bearings with a limited stroke displacement

Fig. 7 Influence of the stroke limit on axial behavior of the spindle.

\subsection{Stick and slip in the preload system}

Frictions occurs during the axial displacement of the rear sleeve in the spindle housing. The stick and slip effect adds a hysteresis on the rear sleeve behavior as shown in Figure 8. The spindle is therefore in a rigid preload configuration when the stick limit is not reached and that the rear sleeve is stuck. When the sleeve is slipping, the preload system is in elastic preload configuration.

Figure 9 illustrates the modeling principles used to model the friction between the rear sleeve and the spindle housing. As radial effort are not considered in this model, the friction can not be modeled with a friction coefficient. An effort $F_{f}$ opposed to the movement direction of the rear sleeve is thus added in the shaft equilibrium equation, representing a simple Coulomb friction force. An extra computation of the model assuming a fixed sleeve is launched to test the slip condition before applying the friction effort.

\subsection{Radial expansion of the bearing rings}

Dynamic effects on the shaft as well as thermal dilatation increase the bearing ring radius. These macroscopic radial expansions change the contact angle $\alpha$ and thus the bearing stiffness. It is modeled by radial displacement of the rings raceway groove curvature center, noted respectively $u_{i}$ and $u_{o}$ for inner and outer ring in the bearing model (see Eq. (1) and Figure 3). For a given bearing initial contact angle and a given steady-state temperature, an initial relative expansion $\Delta u_{0}$ is modeled. The parameter is assumed to remain constant; since the experiments are carried out at iden- 


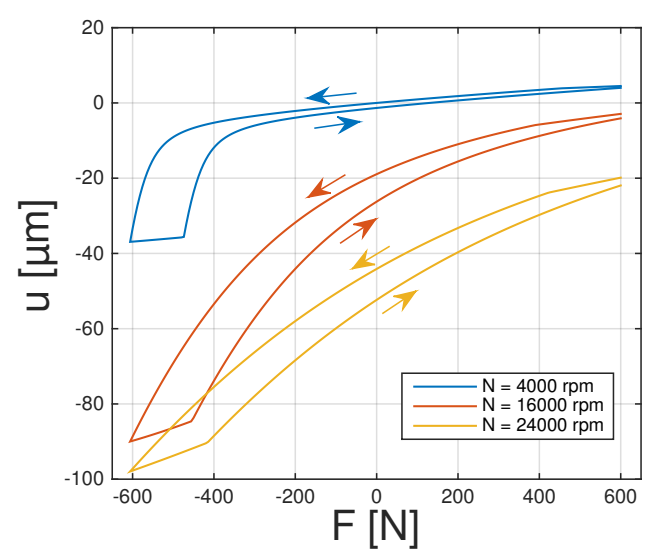

(a) Axial displacement of the shaft

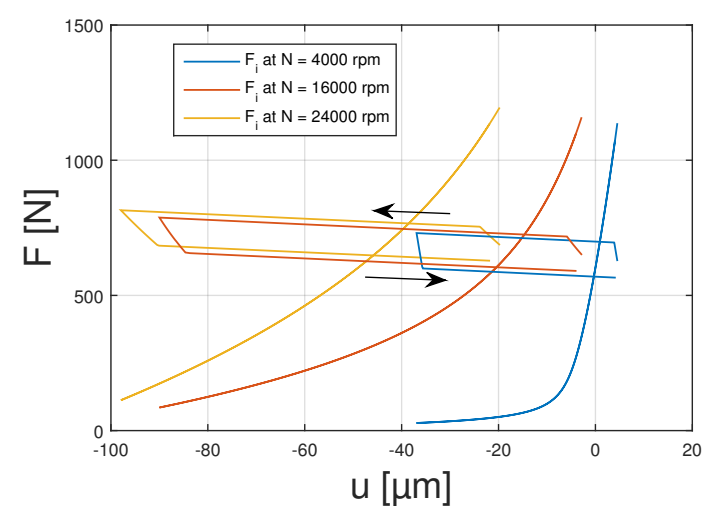

(b) Loads on the bearings

Fig. 8 Influence of the friction on the axial behavior of the spindle.

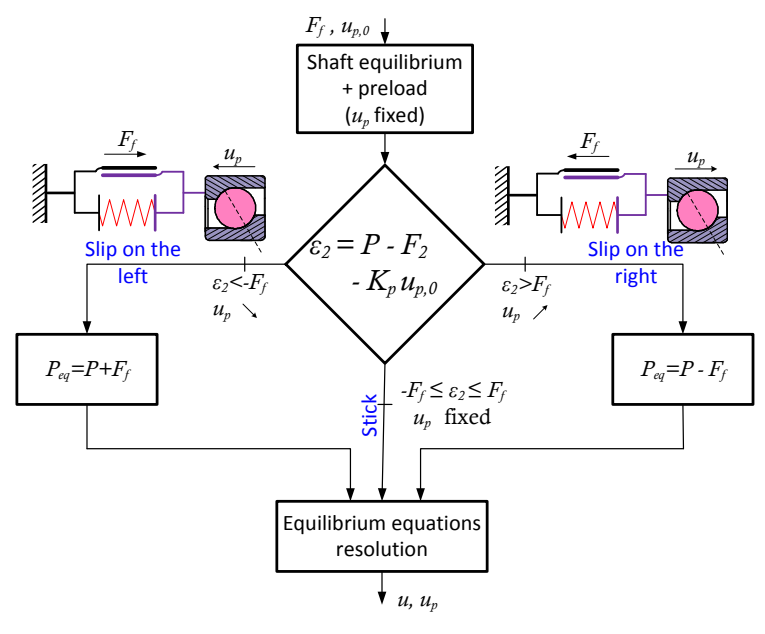

Fig. 9 Principle of the friction modeling.

tical steady-state operating temperature. In addition, the radial expansion due to the centrifugal forces on shaft and inner ring are modeled with a parameter $d$ and varies with the square of the spindle speed. The difference between ring radial expansions at a given speed $N$ is noted $\Delta u_{N}$ and results from :

$\Delta u_{N}=\Delta u_{0}+d \omega^{2}$

Figure 10 shows the influence of radial expansions of the bearing rings on the axial behavior of the shaft. A decrease of the axial stiffness of the shaft is clearly observable at higher speed.

\subsection{Axial shrinkage of the shaft}

The dynamics effects have also an axial influence on the shaft. Indeed, due to the Poisson effect, the centrifugal

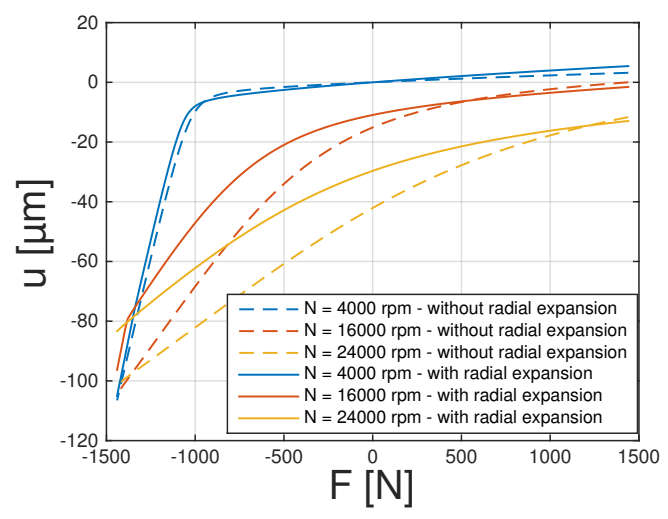

(a) Axial displacement of the shaft

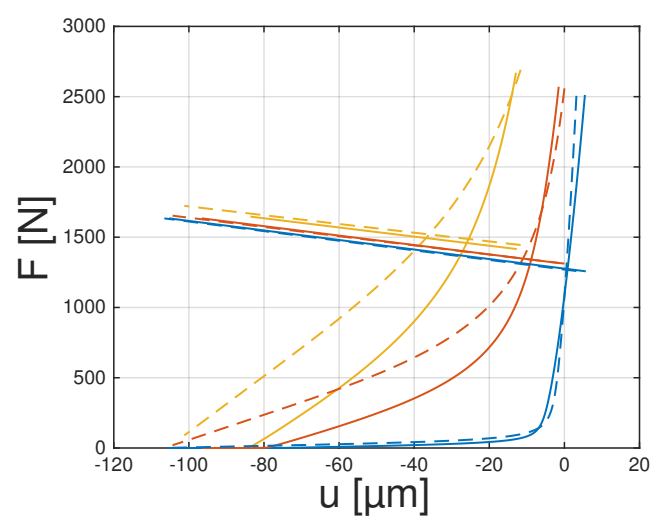

(b) Loads on the bearings

Fig. 10 Radial expansion effect on the axial displacement of the shaft.

radial expansion induces an axial shrinkage of the shaft. The axial deflection $u_{a s_{f}}$ and $u_{a s_{2}}$ of respectively the front side and the rear bearing are added in the model. 
The second term of Eq. (6) becomes :

$\delta_{x 2}=u_{p}-\left(u+u_{a s_{2}}\right)+\delta_{x 2,0} \stackrel{\text { analytical bearing model }}{\longrightarrow} F_{c}$

The final displacement of the front face of the shaft becomes $u+u_{a s_{f}}$. As shown in Figure 11, the axial shrinkage of the shaft decreases the load on the rear bearing at higher speed.

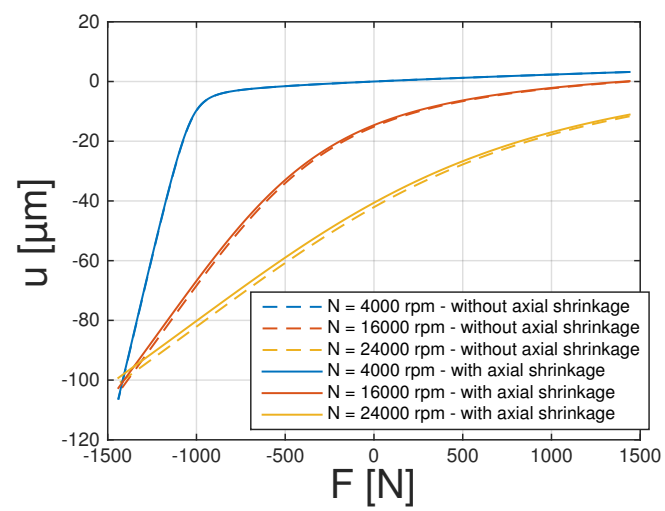

(a) Axial displacement of the shaft

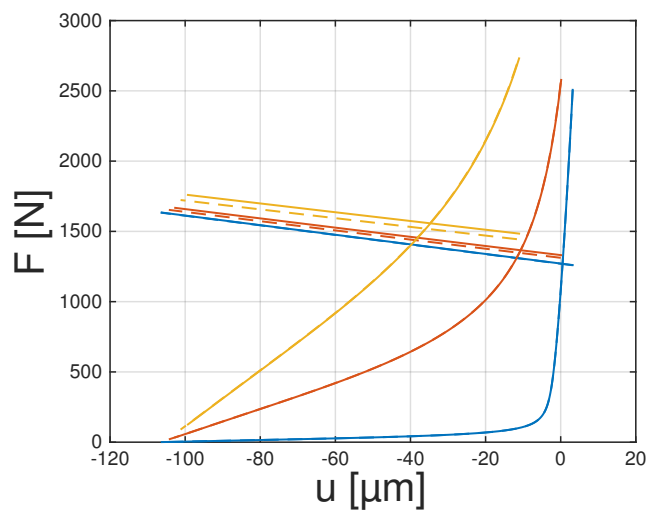

(b) Loads on the bearings

Fig. 11 Axial shrinkage effect on the axial displacement of the shaft.

\section{Experimentation}

\subsection{Experimental Setup}

An original experimental device has been developed to apply bidirectional axial loads to the rotating spindle, see Figure 12. The displacement of the machine tool along the $\mathrm{Z}$ axis generates a pure axial load on the shaft through the spring rings. Radial load and bending moment are avoided thanks to a radial clearance foreseen

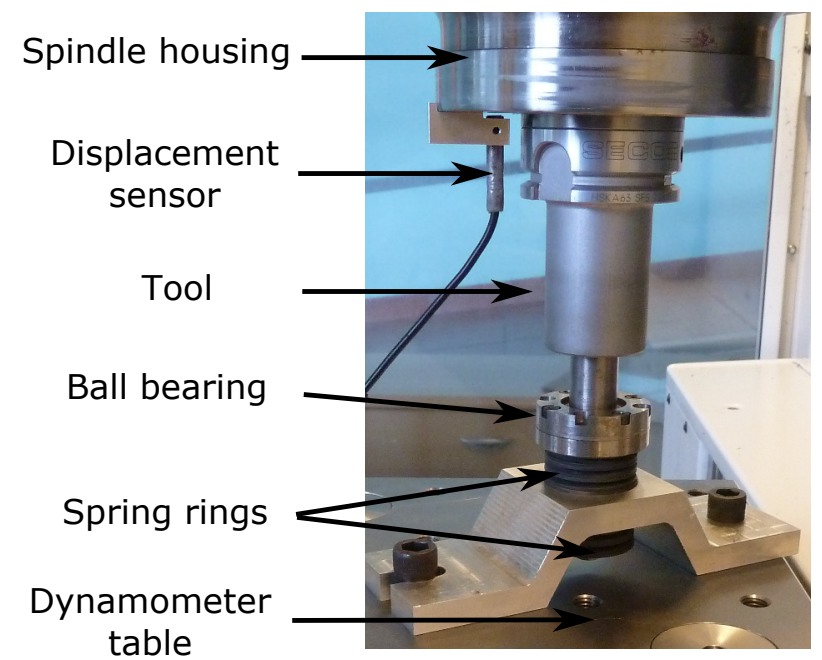

Fig. 12 Experimental device for applying axial load on a rotating spindle.

in the device. A 3-axis 9255B Kistler dynamometer table measures the axial force $F$. An eddy current sensor is used to measure the relative displacement between the shaft and the spindle housing at the front end of the spindle.

A loading cycle of $\pm 1500 N$ is run at different spindle speeds $N$ (from 4000 to $24000 \mathrm{rpm}$ ). Experiments are carried out at an identical steady-state operating temperature and are of short duration to avoid the influence of thermal effects on the results. The sampling frequency is $12.5 \mathrm{kHz}$. It allows to filter axial runout of the surface rotating in front of the sensor. The repeatability of the experiment has been successfully verified.

\subsection{Experimental Results}

The measured signals are filtered with a median filter to keep only the relative quasi-static displacement between the shaft and the spindle housing. Despite fast experiments, a small drift in the displacement measurement can be observed at low speed due to spindle cooling. It can be compensated by linear interpolation between idle rotations before and after the loading cycles. The average displacement from the three measurement cycles are calibrated considering $u=0$ for $F=0$ at 4000 rpm.

Figure 13 shows the results of the measurement for different speed. Three specific zones can be observed on the lower speed curve $(N=4000 \mathrm{rpm})$ :

- Zone A corresponds to the normal behavior of the bearing arrangement with elastic preload.

- Zone $B$ corresponds to a rapid drop in the axial stiffness of the spindle due to the contact loss in the front bearing. It can lead to the approximation of 
the preload value : $P \approx F_{A}=1000 N$. Assuming that the bearing stiffness is much more important than that of the preload springs, the axial stiffness in Zone $\mathrm{B}$ corresponds to the preload springs. $K_{p} \approx$ $\left.\frac{d F}{d u}\right|_{\text {Zone B }}=4 \mathrm{~N} / \mu \mathrm{m}$.

- Zone $C$ starts when the sleeve reaches its stroke limit, which generates an increase in the spindle stiffness. This phenomenon is explained in subsection 3.1.

The hysteresis on the curve is due to friction in the preload system as presented in subsection 3.2.

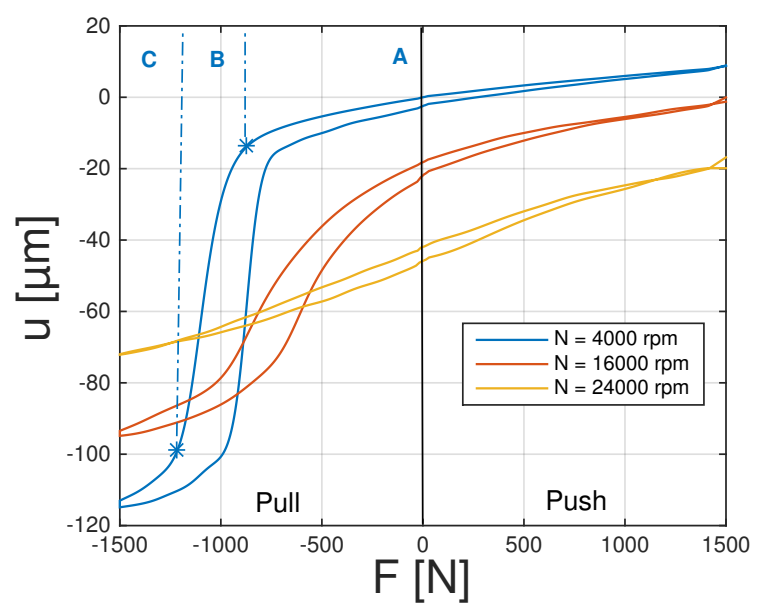

Fig. 13 Experimental results of the displacement $u$ in relation to the axial load $F$ at different speed.

\section{Model enrichment and updating}

This section presents the model updating of the axial model presented in section 2 with the experimental results.

\subsection{Results for a basic model}

Figures 14 shows the axial behavior of the spindle obtained with a basic model. The dynamic effects on bearing balls are considered in this model, but not the phenomenological enrichments introduced in section 3 . It is similar to model of commercial bearing software, when considering identical steady-state operating temperature.

The parameters of the basic model $\left(P\right.$ and $\left.K_{p}\right)$ were updated as presented in subsection 2.2 and Eq. 8. The axial displacement of the shaft is then simulated and compared to the experimental results. It reveals that the basic model is not accurate enough. The axial stiffness between $-500 N<F<500 N$ corresponds to operating conditions and needs to be well predicted by the model. The basic model is therefore too simple to explain the complex behavior of the spindle, even if dynamics effects on the balls are taken into account in the bearing model. The model enrichment methodology presented in section 2.2 is required.

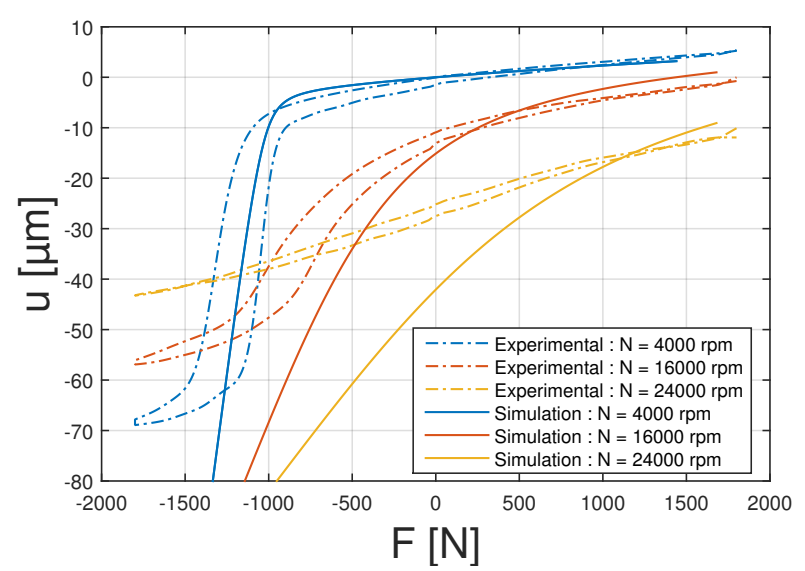

Fig. 14 Axial behavior of a rotor with a basic model.

\subsection{Classification of phenomena significance}

The model enrichment methodology presented in subsection 2.2 was applied to the experimental results (of subsection 4.2), introducing the new phenomena modeled in section 3 . The table 1 shows the results of the sensitivity analysis and of the model updating at the different steps of the model enrichment.

The most sensitive parameters before the first updading were the preload parameters : $P$ and $K_{p}$. They were updated during the initial step, which was the basic model. The results of the initial step, as presented in Figure 14, were not good enough. Then, the second sensitivity analysis $(\kappa)$ revealed that the sleeve parameters (stroke limit and friction) are the most important phenomena on this spindle, after the preload. Due to coupling between the different parameters of each phenomenon, they must be updated at the same time. Therefore, $u_{p, l i m}, \Delta_{s l}$ and $F_{f}$ been updated during step 2. Finally, radial expansions and axial shrinkage were of less importance than the previous considered phenomena, but not negligible to obtain an accurate model. Hence, they were updated in the third steps.

Very small improvement could be achieved with a fourth step, updating the $f_{e}$ parameter of the ball bearing. Indeed, it is the most sensitive of the remaining parameters. However, with an average accuracy of $1.96 \mu \mathrm{m}$, 
Table 1 Results of the sensitivity analysis and of the model updating

\begin{tabular}{|c|c|c|c|c|c|c|c|c|c|c|c|}
\hline & & \multicolumn{2}{|c|}{ range } & \multicolumn{3}{|c|}{ initial step } & \multicolumn{2}{|c|}{ step 2} & \multicolumn{2}{|c|}{ step 3} & \\
\hline \multicolumn{2}{|c|}{ parameter } & $\min$ & $\max$ & nominal & $\kappa[\mu m]$ & updated & $\kappa[\mu m]$ & updated & $\kappa[\mu m]$ & updated & $\kappa[\mu m]$ \\
\hline \multirow{2}{*}{ preload } & $P[N]$ & $\overline{500}$ & 1500 & 950 & 127.3 & 728 & 12.79 & 1174 & 13.02 & 1067 & 441.72 \\
\hline & $K_{p}[N / \mu m]$ & 0.1 & 15 & 2.5 & 383.7 & 8.79 & 18.86 & 1.53 & 12.78 & 1.77 & 36.72 \\
\hline \multirow{2}{*}{$\begin{array}{l}\text { stroke } \\
\text { limit }\end{array}$} & $u_{p . \lim }[\mu m]$ & -1 & -300 & -275 & 17.84 & & 14.39 & -112 & 45.72 & -122 & 25.09 \\
\hline & $\Delta s l[\mu m]$ & 0 & 40 & 0 & 0.0005 & & 1.523 & 25.3 & 7.628 & 37.4 & 0.5502 \\
\hline \multirow{2}{*}{$\begin{array}{l}\text { radial } \\
\text { expansions }\end{array}$} & $\Delta u_{0}[\mu m]$ & -30 & 70 & 0 & 4.827 & & 4.809 & & 7.450 & -24.2 & 0.5301 \\
\hline & $d .10^{6}\left[\mu m . s^{-2}\right]$ & 0 & 60 & 0 & 3.555 & & 3.360 & & 6.644 & 46.0 & 0.2388 \\
\hline friction & $F_{f}[N]$ & 0 & 200 & 0 & 29.39 & & 10.72 & 90.9 & 1.195 & 91.8 & 4.212 \\
\hline \multirow{2}{*}{$\begin{array}{l}\text { axial } \\
\text { shrinkage }\end{array}$} & $a .10^{6}\left[\mu \mathrm{m} . \mathrm{s}^{-2}\right]$ & 0 & 100 & 0 & 2.136 & & 1.123 & & 0.4142 & 6.60 & 0.2691 \\
\hline & $b .10^{6}\left[\mu m . s^{-2}\right]$ & -500 & 0 & 0 & 11.88 & & 3.431 & & 10.88 & 147 & 1.168 \\
\hline \multirow{8}{*}{$\begin{array}{l}\text { ball } \\
\text { bearing }\end{array}$} & $D[\mathrm{~mm}]$ & & $8.73 \pm$ & $1 \%$ & 0.0349 & & 0.0347 & & 0.0552 & & 0.0016 \\
\hline & $d_{m}[m m]$ & & $82.5 \pm$ & $1 \%$ & 0.0132 & & 0.0129 & & 0.0260 & & 0.0002 \\
\hline & $f_{e}$ & & $0.54 \pm$ & $1 \%$ & 1.157 & & 0.1996 & & 2.018 & & 0.1625 \\
\hline & $\nu_{b}$ & & $0.26 \pm$ & & 0.0058 & & 0.0077 & & 0.0074 & & 0.0070 \\
\hline & $\nu_{r}$ & & $0.3 \pm$ & & 0.0029 & & 0.0039 & & 0.0037 & & 0.0035 \\
\hline & $E_{b}[G P a]$ & & $315 \pm$ & & 0.0201 & & 0.0267 & & 0.0255 & & 0.0241 \\
\hline & $E_{r}[G p a]$ & & $210 \pm$ & & 0.0294 & & 0.0391 & & 0.0373 & & 0.0353 \\
\hline & $\rho_{b}\left[\mathrm{~kg} / \mathrm{m}^{3}\right]$ & & $3190 \pm$ & & 0.5527 & & 0.5394 & & 1.088 & & 0.0033 \\
\hline \multicolumn{2}{|c|}{ Mean deviation : $\varepsilon[\mu m]$} & & & 41.4 & & 12.9 & & 6.80 & & 1.96 & \\
\hline
\end{tabular}

the obtained model is precise enough. It clearly explains the complex behavior of the spindle, by a set of simple and uncoupled physical phenomena.

\subsection{Model Updating results}

The results obtained after the third step of enrichment and update are presented on Figure 15. Figure 15(a) shows the comparison of the axial behavior at different spindle speeds, obtained experimentally and with the updated model. The simulations are in good agreement with the experimentation, especially at high speed where there is a need for such advanced model. The results for other speeds that were not used in the updating, have also been compared to the experiment. It was well correlated, which validates the model.

The loads on the bearings are presented on Figure 15 (b). It is interesting to notice that the added phenomena affect significantly the spindle bearings behavior, especially at high speed.

\section{Conclusion}

An original model of the axial behavior of the spindle has been presented. Moreover, an enrichment strategy that consists of several steps of sensitivity analysis and model update have been explained. This work provides a better understanding of the physical phenomena involved in the spindle dynamics at high speed. The modeling of the rear sleeve's behavior, the radial expansions of the bearing rings and the axial shrinkage of the shaft has been detailed as well as their influence on the axial behavior and on the preload evolution. Experimentations to measure the axial behavior of a rotating spindle have been performed with a new specific device and analyzed. The presented model have been updated with the enrichment strategy. The results of the updating obtained before and after the enrichment have been showed. The development of a just accurate enough model have been achieved with the study of the phenomena significance, thanks to sensitivity analysis. An advantage of this methodology is that it does not require a complex thermal model of the spindle to simulate accurately the bearing behavior. Friction between the rear sleeve and the spindle housing, combined to the presence of a limited stroke in the preload system play a crucial role in the axial behavior and have a great influence on the preload state of the spindle, and consequently on the bearing stiffness. The final updated model have been compared to the experiments and it was in a very good agreement.

Acknowledgements The research was conducted within the context of the French FUI project "QuaUsi". The experiment were conducted in collaboration with Europe Technologies and Precise Fischer France. The authors would like to thank these companies.

\section{Nomenclature}

\section{Capital Letters}

$\Re \quad$ radial distance between the bearing axis and the groove curvature center $\Re_{i}=0.5 d_{m}+\left(f_{i}-0.5\right) D \cos \alpha_{0}$

K bearing stiffness matrix 


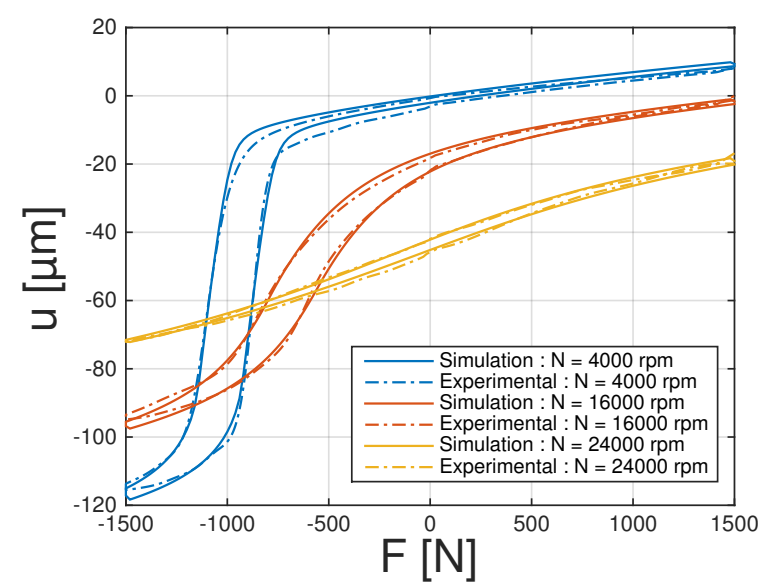

(a) Axial displacement of the shaft

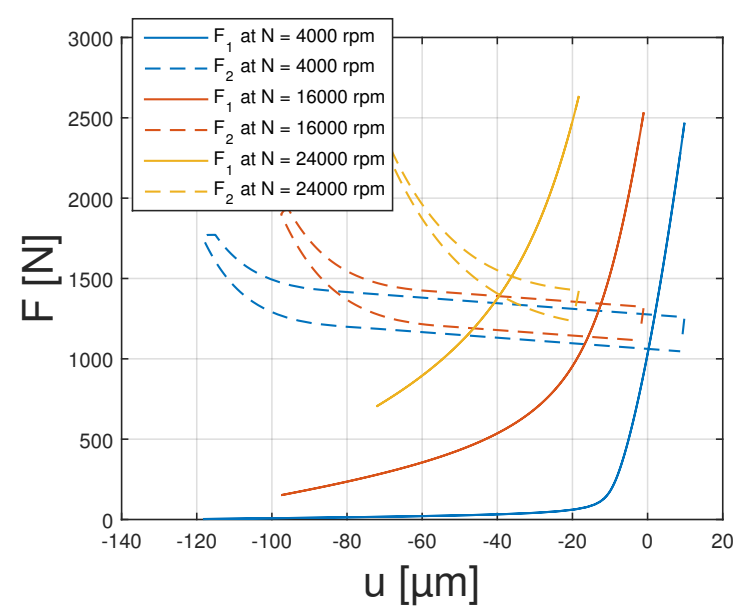

(b) Loads on the front and rear bearings

Fig. 15 Axial behavior of the rotor with the enriched and updated model.

$A_{1}, A_{2}$ axial and respectively radial projection of the distance between groove curvature centers

$B D$ distance between groove curvature centers without load or speed

$D \quad$ ball diameter

$F \quad$ axial load on the shaft

$F_{c} \quad$ centrifugal force on ball

$F_{f} \quad$ Friction force

$K_{p} \quad$ preload springs stiffness

$K_{s l} \quad$ equivalent stiffness applied to model the gradually increasing contact surface between the rear sleeve and the stop

$K_{x x}=K(1,1)$ axial stiffness of the ball bearing

$M_{g} \quad$ ball gyroscopic moment

$N \quad$ shaft speed

$P \quad$ bearing preload

$Q \quad$ ball-raceway normal load

$X_{1}, X_{2}$ axial and radial distance between ball center and groove curvature center of the outer ring

J Jacobian of the axial equilibrium function, Eq. (5)

\section{Greek Symbols}

$\alpha \quad$ contact angle

$\Delta p_{s} \quad$ variation range of parameter $p_{s}$

$\Delta s l$ transition region of the stroke limit

$\Delta u_{N}=u_{i}-u_{o}$ difference between radial ring expansions at speed $N$

$\delta \quad$ ball-raceway normal displacement

$\kappa_{s} \quad$ sensitivity of the parameter $p_{s}$

$\lambda$ distribution parameter for the ball gyroscopic moment between rings

$\psi \quad$ ball angular position on the pitch circle

$\varepsilon \quad$ mean deviation between experiment and simulations

$\zeta \quad$ perturbation factor of the variation range

\section{Lowercase Letters}

d global displacement of the inner ring

f global load on inner ring

$\mathbf{u}$ axial displacement of the shaft

$d_{m} \quad$ ball orbital diameter

$f \quad=r / D$

$p_{s} \quad$ model parameter

$r \quad$ raceway groove curvature radius

$u_{i}, u_{o}$ radial displacement of groove curvature centers

$u_{a s_{f}}, u_{a s_{2}}$ axial deflections respectively of the front face and the back bearing due to centrifugal effects on the shaft

$u_{p, l i m}$ stroke limit of the rear sleeve

Subscripts

$i \quad$ inner ring

$o \quad$ outer ring

\section{References}

1. Abele E, Altintas Y, Brecher C (2010) Machine tool spindle units. CIRP Annals - Manufacturing Technology 59:781-802, DOI 10.1016/j.cirp.2010.05.002

2. Bossmanns B, Tu JF (1999) A thermal model for high speed motorized spindles. International Journal of Machine Tools and Manufacture 39(9):13451366, DOI 10.1016/S0890-6955(99)00005-X

3. Cao H, Holkup T, Altintas Y (2011) A comparative study on the dynamics of high speed spindles with respect to different preload mechanisms. The International Journal of Advanced Manufacturing Technology 57(9-12):871-883, DOI 10.1007/s00170-0113356-9

4. Cao H, Niu L, He Z (2012) Method for vibration response simulation and sensor placement optimiza- 
tion of a machine tool spindle system with a bearing defect. Sensors (Switzerland) 12(7):8732-8754, DOI $10.3390 / \mathrm{s} 120708732$

5. Cao Y, Altintas Y (2004) A General Method for the Modeling of Spindle-Bearing Systems. Journal of Mechanical Design 126(6):1089, DOI $10.1115 / 1.1802311$

6. Chen JS, Hwang YW (2006) Centrifugal force induced dynamics of a motorized high-speed spindle. International Journal of Advanced Manufacturing Technology 30(1-2):10-19, DOI 10.1007/s00170005-0032-y

7. De Castelbajac C, Ritou M, Laporte S, Furet B (2014) Monitoring of distributed defects on HSM spindle bearings. Applied Acoustics 77:159-168, DOI 10.1016/j.apacoust.2013.07.008

8. Ertürk A, Özgüven H, Budak E (2007) Effect analysis of bearing and interface dynamics on tool point FRF for chatter stability in machine tools by using a new analytical model for spindle-tool assemblies. International Journal of Machine Tools and Manufacture 47(1):23-32, DOI 10.1016/j.ijmachtools.2006.03.001

9. Gagnol V, Bouzgarrou B, Ray P, Barra C (2007) Model-based chatter stability prediction for highspeed spindles. International Journal of Machine Tools and Manufacture 47(7-8):1176-1186, DOI 10.1016/j.ijmachtools.2006.09.002

10. Gao S, Cheng K, Ding H, Fu H (2016) Multiphysicsbased design and analysis of the high-speed aerostatic spindle with application to micro-milling. Proceedings of the Institution of Mechanical Engineers, Part J: Journal of Engineering Tribology 230(7):852-871, DOI 10.1177/1350650115619609

11. Holkup T, Hol \'y S (2005) Prediction of Thermal Stability of Spindle Bearing Systems. In: Proceedings of the 4th YSESM Youth Symposium of Experimental Solid Mechanics, pp 107-108

12. Houpert L (1997) A uniform analytical approach for ball and roller bearings calculations. Journal of Tribology 119(4):851-859, DOI 10.1115/1.2833896

13. Jedrzejewski J, Kwasny W (2010) Modelling of angular contact ball bearings and axial displacements for high-speed spindles. CIRP Annals Manufacturing Technology 59(1):377-382, DOI 10.1016/j.cirp.2010.03.026

14. Jones AB (1960) A General Theory for Elastically Constrained Ball and Radial Roller Bearings Under Arbitrary Load and Speed Conditions. Journal of Basic Engineering 82(2):309, DOI $10.1115 / 1.3662587$

15. Li H, Shin YC (2004) Analysis of bearing configuration effects on high speed spindles using an integrated dynamic thermo-mechanical spindle model. International Journal of Machine Tools and Manufacture 44(4):347-364, DOI 10.1016/j.ijmachtools.2003.10.011

16. Lin CW, Tu JF, Kamman J (2003) An integrated thermo-mechanical-dynamic model to characterize motorized machine tool spindles during very high speed rotation. International Journal of Machine Tools and Manufacture 43(10):1035-1050, DOI 10.1016/S0890-6955(03)00091-9

17. Lynagh N, Rahnejat H, Ebrahimi M, Aini R (2000) Bearing induced vibration in precision high speed routing spindles. International journal of machine tools and manufacture 40(4):561-577, DOI DOI: 10.1016/S0890-6955(99)00076-0

18. Noel D, Le Loch S, Ritou M, Furet B (2013) HSM Spindle Model Updating With Physical Phenomena Refinements. In: Volume 7A: 9th International Conference on Multibody Systems, Nonlinear Dynamics, and Control, ASME, p V07AT10A035, DOI 10.1115/DETC2013-13141

19. Noel D, Ritou M, Furet B, Le Loch S (2013) Complete Analytical Expression of the Stiffness Matrix of Angular Contact Ball Bearings. Journal of Tribology 135(4):041,101, DOI 10.1115/1.4024109

20. Ozturk E, Kumar U, Turner S, Schmitz T (2012) Investigation of spindle bearing preload on dynamics and stability limit in milling. CIRP Annals - Manufacturing Technology 61(1):343-346, DOI 10.1016/j.cirp.2012.03.134

21. Palmgren A (1959) Ball and roller bearing Engineering, 3rd edn. SKF industries, Philadelphia

22. Rantatalo M, Aidanpää JO, Göransson B, Norman P (2007) Milling machine spindle analysis using FEM and non-contact spindle excitation and response measurement. International Journal of Machine Tools and Manufacture 47(7-8):1034-1045, DOI 10.1016/j.ijmachtools.2006.10.004

23. Spiewak S, Nickel T (2001) Vibration based preload estimation in machine tool spindles. International Journal of Machine Tools and Manufacture 41(4):567-588, DOI 10.1016/S0890-6955(00)00081$\mathrm{X}$

24. Swanson E, Powell CD, Weissman S (2005) A practical review of rotating machinery critical speeds and modes. Sound and Vibration 39(5):10-17

25. Yamazaki T, Matsubara A, Ikenaga S (2012) Measurement of rigidity change of preload switching spindle. International Journal of Automation Technology 6(2):175-179

26. Zivkovic A, Zeljkovic M, Tabakovic S, Milojevic Z (2014) Mathematical modeling and experimental testing of high-speed spindle behavior. The Inter- 
national Journal of Advanced Manufacturing Technology DOI 10.1007/s00170-014-6519-7

27. Zverv I, Pyoun YS, Lee KB, Kim JD, Jo I, Combs A (2005) An elastic deformation model of high speed spindles built into ball bearings. Journal of Materials Processing Technology 170(3):570-578, DOI 10.1016/j.jmatprotec.2005.05.038

\section{A Jacobian used in the solving algorithm considering the stroke limit}

$\mathbf{J}=\left[\begin{array}{cc}-K_{x x 1}-K_{x x 2} & K_{x x 2} \\ K_{x x 2} & -\frac{\partial K_{s l} u_{p}}{\partial u_{p}}-K_{p}-K_{x x 2}\end{array}\right]$

with :

$$
\begin{aligned}
\frac{\partial K_{s l} u_{p}}{\partial u_{p}} & =\tan \left(\frac{\pi}{2}\left(\frac{u_{p}-u_{p, l i m}}{\Delta_{s l}}-1\right)\right)^{2} \\
& +\frac{\pi u_{p}}{\Delta_{s l}} \tan \left(\frac{\pi}{2}\left(\frac{u_{p}-u_{p, l i m}}{\Delta_{s l}}-1\right)\right) \cos \left(\frac{\pi}{2}\left(\frac{u_{p}-u_{p, l i m}}{\Delta_{s l}}-1\right)\right)^{-2}
\end{aligned}
$$

